\title{
50 Endurance training and heart rate
}

The resting heart rate is defined as the heart rate measured immediately after a night's rest, while still lying down. In adults, it is approximately 70 beats per minute. Endurance training lowers the heart rate to around 60 beats per minute in most recreational sports enthusiasts, 50 beats per minute in amateur athletes and often as low as 35-40 beats per minute in high-performance athletes. High body temperature (fever) increases the heart rate. a one degree increase in body temperature causes ten extra heartbeats. Measuring your resting heart rate is therefore a useful tool to check on your general health status. If your resting pulse is raised by more than 8 beats per minute in the morning, your exercise routine should be reduced or completely stopped.

The optimal heart rate during endurance exercise is between 60 and $85 \%$ of the maximum achievable heart rate.

In healthy persons, the maximum heart rate achieved during physical activity is age-dependent (Larson et al. 2013). It is approximately calculated by 220 minus age. Any personal training program should be based on this calculation.

This equates to walking at a fast pace while still being able to talk without difficulty. The correlation between heart rate and degree of physical activity is nearly linear at a heart rate range of around $120-170$, which is useful when devising training programs.
It is sometimes useful to check your heart rate during physical activity. Ideally, this should be measured using small devices attached to the body. Heart rate measurements checked manually have the disadvantage that physical activity must be interrupted, limiting accuracy. It takes several seconds to accurately check your pulse when resting, during which time it will have already slowed down.

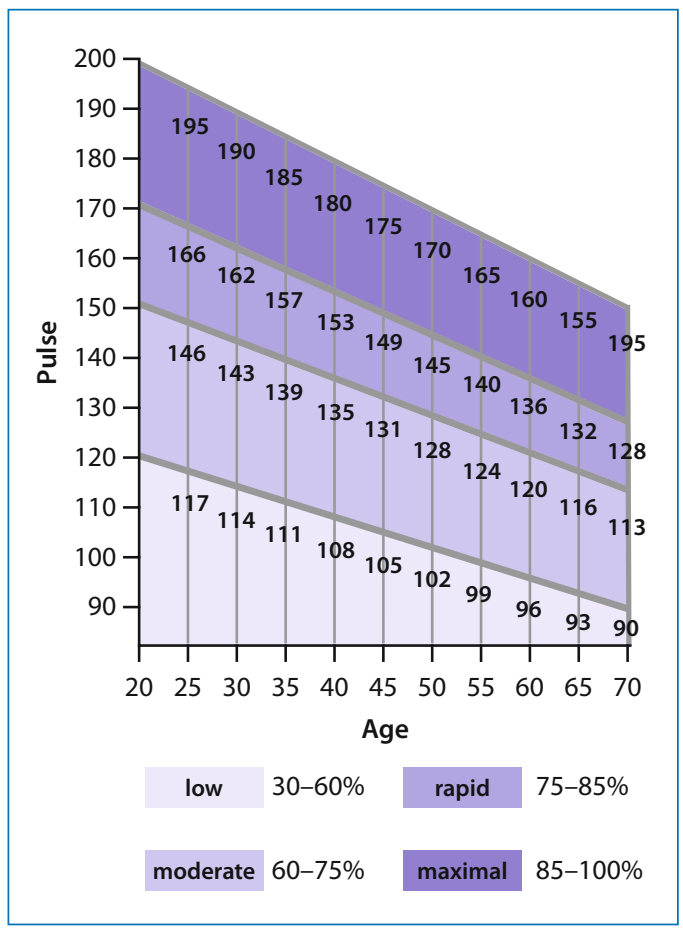

- Fig. 50.1 Relation of workout intensity and pulse for different age groups 